\title{
Intermolecular forces and fixed-node diffusion Monte Carlo: A brute force test of accuracies for $\mathrm{He}_{2}$ and $\mathrm{He}-\mathrm{LiH}$
}

\author{
Massimo Mella ${ }^{a}$ \\ Dipartimento di Chimica Fisica ed Elettrochimica, Universita' degli Studi di Milano, via Golgi 19, \\ 20133 Milano, Italy \\ James B. Anderson ${ }^{\text {b) }}$ \\ Department of Chemistry, The Pennsylvania State University, University Park, Pennsylvania 16802
}

(Received 8 July 2003; accepted 31 July 2003)

\begin{abstract}
The accuracy of the fixed-node approximation and diffusion Monte Carlo method in computing the interaction energy of van der Waals systems was investigated. Tests were carried out by simulating the electronic structures of $\mathrm{He}_{2}$ and $\mathrm{He}-\mathrm{LiH}$. These two systems were chosen as representative of two fundamentally different interactions, namely the weak dispersion forces in $\mathrm{He}_{2}$ and the dipole/ induced-dipole interaction in $\mathrm{He}-\mathrm{LiH}$. The results for both systems are in excellent agreement with "state of the art" calculations, thereby indicating a high accuracy for the fixed-node approximation. Also, our interaction energies for $\mathrm{He}-\mathrm{LiH}$ indicate that the coupled cluster single double triple method [Taylor and Hinde, J. Chem. Phys. 111, 973 (1999)] gives an accurate prediction of the interaction potential for that system. (C) 2003 American Institute of Physics.
\end{abstract}

[DOI: $10.1063 / 1.1612479]$

The accurate description of interaction potentials is of paramount importance in rationalizing and predicting the physical chemistry of gas phase reactions, molecular clusters, and bulk systems. For such descriptions quantum chemists have developed various approaches delivering different levels of sophistication and predictive power. These methods usually fall into one or the other of two conceptual families, supermolecular or perturbative. In the supermolecular approach one computes the interaction potential between two or more chemically relevant fragments by subtracting the energy of the separate species from the energy of the supersystem, while in the perturbative approach one exploits the fact that only small changes in the electronic structures of the fragments are induced by their mutual interaction.

However, independent of the method of choice, two main difficulties are invariabily encountered. These are (1) the necessity of introducing the electronic correlation in the description of the molecular forces and (2) the dependence of the predicted forces on the quality of the basis set employed. Although one could always circumvent the first difficulty by selecting a correlated method such as Møller-Plesset $(\mathrm{MP} n)$, coupled cluster $(\mathrm{CC})$, or one of the variants of the explicit electron interaction family (R12), ${ }^{1}$ the issue of the basis set dependence of the results is quite complicated and delicate. While on one hand there is the need to use the largest possible basis set in order to describe the subtle changes in the electronic structure of the fragments, on the other hand one is faced with the issue of the computational effort required to carry out the calculation. Usually, the more

\footnotetext{
a) Present address: Central Chemistry Laboratory, Department of Chemistry, University of Oxford, South Parks Road, Oxford OX1 3QH, United Kingdom; Electronic mail: Massimo.Mella@chem.ox.ac.uk

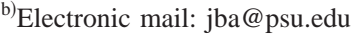

accurate the computational method the higher the computational effort for the basis set.

To make the issue even more complicated, the finite size of the basis set invariabily produces an overestimate of the interaction energy with respect to the complete basis set limit when size-consistent methods are employed. This error is usually called "basis set superposition error" $(\mathrm{BSSE})^{2}$ and it is generated by one of the fragments using the basis set of the other one to stabilize itself. ${ }^{3}$ Although various schemes have been proposed to reduce or eliminate this problem in the framework of the standard quantum chemistry approaches ${ }^{4-6}$ no definitive solution to the problem is currently available for large systems. It is this that has led us in the direction of developing and testing alternative methods for predicting accurate potential energy surfaces for intermolecular interactions.

Among the possible alternatives, the different variants of quantum Monte Carlo $(\mathrm{QMC})^{7}$ used in electronic structure calculations possess distinctive advantages with respect to the more frequently employed ab initio methods. We specialize in describing the diffusion Monte Carlo method (DMC) ${ }^{8-11}$ but similar remarks can be made for the other members of the family. First, by using the position representation in configurational space the DMC method employs a complete basis set. Thus, it is BSSE free. Second, electronic correlation is directly introduced in the treatment by simulating the exact Born-Oppenheimer electronic Hamiltonian without referring to any model system, wave function or Hamiltonian as the starting point. This avoids the slow convergence toward the exact energy upon improving the quality of the basis set which is typical of CC and CI. Third, given any accurate, although approximate, description of a system, DMC always improves the average energy in a sizeconsistent way. 
Using both DMC or Green's function Monte Carlo (GFMC), a method closely related to DMC, highly accurate results have been obtained for $\mathrm{He}_{2},{ }^{12-14} \mathrm{HeH},{ }^{16} \mathrm{He}_{3},{ }^{17,18}$ $\mathrm{H}_{3},{ }^{19,20} \mathrm{H}_{2}^{-}$, and $\mathrm{H}-\mathrm{PsH}{ }^{21}$ The results of these calculations represent the most accurate values to date for the interaction energy of the aforementioned systems. In fact, "exact" QMC solutions with no extrapolations, no interpolations, and no corrections are available for many of these. This suggests that the QMC methods have the potential to contribute to the field of intermolecular forces for larger systems.

Whereas GFMC can be made exact by using a cancellation procedure to sample the ground state of small fermionic systems, the DMC method usually relies on the fixed-node (FN) approximation to satisfy the antisimmetry requirement for an electronic wave function. ${ }^{9}$ This implies that the computed energy is an upper bound of the exact energy of the system. ${ }^{10}$ However, the weak interaction due to the van der Waals force is usually long range. For instance, the $\mathrm{He}_{2}$ equilibrium distance is 5.6 bohr. This large distance makes the overlap between the electron density of the two interacting systems very small so that the nodal surfaces of the wave function for the two interacting fragments may be almost identical to the one obtained by multiplying the wave function of the two isolated systems. To be more specific, let us assume $\Psi_{T}^{(a)}$ and $\Psi_{T}^{(b)}$ to be the two antisymmetric trial functions for the fragments $a$ and $b$. A simple antisymmetric trial wave function for the interacting systems could be written as

$$
\Psi_{T}^{(a b)}=A\left[\Psi_{T}^{(a)} \Psi_{T}^{(b)}\right]=\sum_{P}(-)^{p} P\left[\Psi_{T}^{(a)} \Psi_{T}^{(b)}\right]
$$

where the antisymmetrizer $A$ contains only permutation operators $P$ that exchange the electrons of $a$ with the electrons of $b$ including the identity operator $I$. The net effect of a single use of the operator $P$ can be visualized as the movement of the $\Psi_{T}^{(a)} \Psi_{T}^{(b)}$ product to a different region of the electronic configuration space. This movement can place the centroid of $\Psi_{T}^{(a)} \Psi_{T}^{(b)}$ and $P\left[\Psi_{T}^{(a)} \Psi_{T}^{(b)}\right]$ far away from each other if the distance between the center of mass of $a$ and $b$ is large. Since $\Psi_{T}^{(a)}$ and $\Psi_{T}^{(a)}$ decay exponentially with the distance between an electron and the center of mass, the contribution due to the permuted term to the value, and hence its effect on the node location, of $I\left[\Psi_{T}^{(a)} \Psi_{T}^{(b)}\right]$ should be expected to be small. If this is exactly the case, one would also expect to achieve an accurate nodal error cancellation between the noninteracting fragments and the supermolecular complex. This should also occur if the two wave functions $\Psi_{T}^{(a)}$ and $\Psi_{T}^{(b)}$ are relaxed at the interaction geometry, especially if the systems are different.

Since the above-presented arguments indicate FN-DMC is a possible candidate for accurate predictions of van der Waals interaction energies, we feel it is worth to test this approach on small and medium systems in order to better understand its applicability and limitations.

As a first test case, the helium dimer $\mathrm{He}_{2}$ in its equilibrium geometry ( $\left.R_{\text {eq }}=5.6 \mathrm{bohr}\right)$ for the ground state is chosen. The interaction potential of this system was intensively investigated both for its importance in low temperature condensed matter and cluster physics and as a prototype of a
TABLE I. FN-DMC interaction energy of $\mathrm{He}_{2}$ at $R_{e q}=5.6 \mathrm{bohr}$. Energies in $\mathrm{K}$ and time steps $\tau$ in a.u. $\sigma$ indicates the statistical error of the results.

\begin{tabular}{cll}
\hline \hline$\tau$ & $E(\tau)$ & \multicolumn{1}{c}{$\sigma$} \\
\hline GFMC $^{\mathrm{a}}$ & -10.998 & 0.005 \\
0.000 & -11.07 & 0.08 \\
0.007 & -11.08 & 0.11 \\
0.010 & -11.16 & 0.11 \\
0.012 & -11.10 & 0.10 \\
0.015 & -11.13 & 0.10 \\
\hline
\end{tabular}

${ }^{\mathrm{a}}$ Reference 15 .

system with van der Waals forces. The calculation of the potential curve with an accuracy of better than $0.1 \%$ has been a long-standing goal of modern quantum chemistry (see the list of modern references in Ref. 14) recently met with "exact" QMC calculations. 15

Since the ground state of the He atom is nodeless, the DMC method would give the exact result for the energy of separated atoms. However, instead of using DMC to compute the total energy of He, we rely on the alternate calculations presented in Ref. 22, -2.903724 377034 hartree, for this value.

The trial wave function chosen to guide the simulation for $\mathrm{He}_{2}$ and compute the value of the energy is written as an antisymmetrized product of two Hylleraas-type wave functions multiplied by a many-body Jastrow factor containing dipole-dipole and similar cross terms. ${ }^{23}$ This function has a variational total energy of $-5.807484(2)$ hartree and an estimate root-mean-square fluctuation of the local energy equal to 0.0015 hartree. This model wave function has already been employed to compute the $\mathrm{He}_{2}$ interaction energy curve by means of the GFMC method including exact cancellation. ${ }^{12}$ Using a target population of 5000 walkers, the difference between the FN-DMC energies computed at various time steps $\tau$ and twice the ground state energy of He are shown in Table I. Each calculation required roughly $2600 \mathrm{~h}$ of CPU time on an SP2 machine of the Center for Academic Computing of the Pennsylvania State University. Table I also presents the extrapolated $\tau=0$ value, $-11.07(8) \mathrm{K}$, obtained by fitting the finite $\tau$ results with a linear function, and the most recent GFMC value, ${ }^{15}-10.998(5)$ K. Being based on the sampling of the exact Green's function, the latter does not require extrapolation of any sort. As evident, the FN$\operatorname{DMC}(\tau=0)$ result is in accurate agreement with the GFMC value, differing from the last one only by $0.07(8) \mathrm{K}$. Both values are also in accurate agreement with the most recent $a b$ initio estimates of the same quantity, namely $-10.947 \mathrm{~K}$ (a variational upper bound), ${ }^{24}-10.978 \mathrm{~K},{ }^{25}-11.00 \mathrm{~K},{ }^{26}$ $-10.947 \mathrm{~K},{ }^{27}-11.02 \mathrm{~K},{ }^{28}-10.95 \mathrm{~K},{ }^{29}$ and $-11.059 \mathrm{~K}^{30}$ This suggests that the nodal surfaces are quite accurate for this system.

Motivated by the accurate results obtained for the $\mathrm{He}$ dimer, we applied the FN-DMC method to another test case. A particularly interesting and useful one is that of the interaction between the $\mathrm{He}$ atom and the $\mathrm{LiH}$ molecule. State-tostate cross sections for collisions between these two species are important in modeling the energy transfer in outer space. ${ }^{31}$ Further, the interaction energy between these two 
TABLE II. VMC total energies and root-mean-square fluctuation $\sigma$, FN-DMC total energies, DMC [ $\triangle \mathrm{E}(\mathrm{DMC})]$ and $\operatorname{CCSD}(\mathrm{T})$ interaction energies for $\mathrm{He}-\mathrm{LiH}$ at various geometries. Total energies and flustuations in hartree and interaction energies in $\mathrm{cm}^{-1}$. Distances in bohr. A positive value for $R_{\mathrm{HeCM}}$ indicates that $\mathrm{He}$ is located at the $\mathrm{Li}$ end of $\mathrm{LiH}$.

\begin{tabular}{lccccr}
\hline \hline$R_{\mathrm{HeCM}}$ & $E(\mathrm{VMC})$ & $\sigma$ & $E(\mathrm{DMC})$ & $\Delta E(\mathrm{DMC})$ & \multicolumn{1}{c}{$\mathrm{CCSD}(\mathrm{T})^{\mathrm{a}}$} \\
\hline+5.50 & $-10.95983(15)$ & $0.369(7)$ & $-10.974086(41)$ & $-64(9)$ & -76.08 \\
+4.25 & $-10.96087(14)$ & $0.359(8)$ & $-10.974610(65)$ & $-179(14)$ & -176.59 \\
+3.50 & $-10.95646(15)$ & $0.359(7)$ & $-10.972511(65)$ & $+282(14)$ & +312.51 \\
-7.25 & $-10.94794(19)$ & $0.459(9)$ & $-10.972343(66)$ & $+318(14)$ & +337.52 \\
$\infty$ & & & $-10.973800(66)$ & 0 & \\
\hline \hline
\end{tabular}

${ }^{\mathrm{a}}$ Reference 33 .

fragments has already been investigated by using spin coupled-valence bond (SC-VB) theory ${ }^{32}$ and coupled cluster single double triple $\operatorname{CCSD}(\mathrm{T})$ calculations. ${ }^{33}$ An interesting feature of the interaction potential is the presence of two different minima, one as the $\mathrm{He}$ atom approaches $\mathrm{LiH}$ at the $\mathrm{Li}$ end of the molecule and another as the He atom approaches the $\mathrm{H}$ end of the molecule. Each minimum corresponds to a collinear configuration. These minima are interpreted as deriving from a relatively strong dipole/induceddipole interaction between the dipolar $\mathrm{LiH}$ and the weakly polarizable He. The minimum at the $\mathrm{Li}$ end of the molecule is predicted to be deeper and to lie at shorter distance than the one at the $\mathrm{H}$ end. This behavior can be rationalized by the strong ionic character of the $\mathrm{LiH}$ molecule which is accurately described as $\mathrm{Li}^{+} \mathrm{H}^{-}$at the equilibrium distance. Thus, while approaching the $\mathrm{Li}$ end of the molecule an $\mathrm{He}$ atom can experience the bare molecular field even at short distance due to the compact electronic structure of the $\mathrm{Li}^{+}$ion. Conversely, the electronic distribution on the $\mathrm{H}$ end is quite diffuse due to the strong $\mathrm{H}^{-}$character. So, the repulsive interaction due to the electron overlap starts early and reduces the binding interaction energy at long distance. Although producing overall similar interaction potentials, the SC-VB and $\operatorname{CCSD}(\mathrm{T})$ methods differ in predicting the relative location and magnitude of the two minima. More specifically, the SC-VB procedure predicts interaction energies only about one-third of those predicted by $\operatorname{CCSD}(\mathrm{T})$ as well as somewhat larger equilibrium separations. These quantitative discrepances give rise to qualitatively different results for the ground state of the $\mathrm{LiH}-\mathrm{He}_{n}$ complexes. Whereas the $\operatorname{CCSD}(\mathrm{T})$ potential predicts binding even for $\mathrm{He}-\mathrm{LiH},{ }^{34}$ nuclear DMC simulations using the SC-VB potential energy surface (PES) suggest $\mathrm{LiH}-\mathrm{He}_{n}$ to dissociate into $\mathrm{LiH}$ and $\mathrm{He}_{n} \cdot{ }^{35}$ Differences in the state-to-state cross section in the inelastic scattering of $\mathrm{He}$ from $\mathrm{LiH}$ are also seen. ${ }^{36}$

We carried out FN-DMC calculations for four different geometries of the interacting supermolecular complex He$\mathrm{LiH}$. The $\mathrm{LiH}$ bond distance was fixed at the equilibrium value for the isolated molecule (3.01397 bohr), and a simulation was carried out to estimate the LiH total energy obtaining $E=-8.070075(66)$ hartree. This value compares well with the lowest-energy variational result, -8.070449 hartree. ${ }^{37}$ Using the latter it is possible to obtain an estimate of the LiH fixed-node error for the DMC simulation, namely $0.000374(66)$ hartree. All the DMC simulations employed the time step size $\tau=0.001$ a.u. and a walker ensemble com- posed of 5000 configurations. These parameter values represent in our experience an adequate choice to reduce the combined time step and population bias in the calculated energies to a values smaller than the statistical error in the energies. After the equilibration phase, each simulation consumed roughly $240 \mathrm{~h}$ of CPU time on a two year old single processor Pentium III $800 \mathrm{MHz}$.

As a trial wave function for both $\mathrm{LiH}$ and $\mathrm{He}-\mathrm{LiH}$ we employed the widely used expression written as a determinant times a Jastrow factor. ${ }^{38}$ The molecular orbitals used in the determinantal part of the trial wave function were obtained using the GAUSSIAN 98 suite $^{39}$ and the restricted Hartree-Fock procedure. The Slater type orbital basis set for $\mathrm{Li}$ and $\mathrm{H}$ was taken from the work by Liu et al. ${ }^{40}$ on the $\mathrm{LiH}^{-}$anion, while the HF basis set for He from Ref. 41 was supplemented with a $2 p$ function whose exponent was optimized using MP2 calculations. The Slater basis set was approximated as a linear combination of Gaussian orbitals (either STO-10G or STO-8G) in all the GAUSSIAN 98 calculations. The Jastrow part of the trial function was optimized by using a robust estimator approach recently introduced. ${ }^{42}$ The variational results of the optimization procedure for the total energy $E(\mathrm{VMC})$ and the root-meansquare fluctuation of the local energy $\sigma$ are presented in Table II. Comparing the fluctuation of the local energy for these wave functions with the one of the $\mathrm{He}-\mathrm{He}$ trial wave function, it can be clearly seen that the latter is orders of magnitude more accurate than the ones derived by the "Jastrow times determinant" model.

The DMC results obtained using the optimized trial wave functions for the $\mathrm{He}-\mathrm{LiH}$ systems are shown in Table II. In the table, $R_{\mathrm{HeCM}}$ represents the distance between the $\mathrm{He}$ atom and the $\mathrm{LiH}$ center of mass. The He atom lies on the $\mathrm{LiH}$ bond axis, and a positive sign of $R_{\mathrm{HeCM}}$ indicates it is located at the $\mathrm{Li}$ end of the molecule. The system geometries were chosen to probe different parts of the potential surface, namely the bottom of the well of the strong $\mathrm{He}-\mathrm{Li}^{+}$interaction and the repulsive walls at both ends. Together with the DMC results we show also the interaction energy obtained by using counterpoise corrected $\operatorname{CCSD}(\mathrm{T})$ calculations. ${ }^{33}$ Comparing the values in Table II, it may be seen that the two different techniques produce good agreement with each other. This is especially true for $R_{\mathrm{HeCM}}=+4.25$ and +5.50 bohr, points that are located inside the attractive well of the potential. Despite their uncertainties, the DMC results for $R_{\mathrm{HeCM}}=+3.50$ and -7.25 bohr seem to indicate that the 
repulsive part of the $\operatorname{CCSD}(\mathrm{T})$ potential is steeper than the DMC one. A direct comparison of the DMC results with $\mathrm{SC}-\mathrm{VB}$ results cannot be made because of differing geometries. However, in Ref. 36 a comparison between CCSD(T) and $\mathrm{SC}-\mathrm{VB}$ results was carried out for geometries that only slightly differ from the ones employed in the present work. The results highlighted the much less attractive behavior of the SC-VB potential with respect the $\operatorname{CCSD}(\mathrm{T})$ one, roughly a factor of 3 in terms of the well depth, and hence also with respect to the DMC results.

In summary, in this work we used the FN-DMC method to compute accurate values for the interaction energies of $\mathrm{He}-\mathrm{He}$ and $\mathrm{He}-\mathrm{LiH}$. The computed values are in excellent agreement with those of prior "state of the art" electronic structure calculations available for these systems. This suggests that the fixed-node approximation does not introduce any important bias in FN-DMC calculations for these and similarly interacting systems. In using the FN-DMC method, we were able to confirm the accuracy of prior $\operatorname{CCSD}(\mathrm{T})$ calculations for $\mathrm{He}-\mathrm{LiH}$.

We note that the computational effort required for a $\mathrm{FN}$ DMC calculation to reach a chosen statistical accuracy in the energy is expected to scale as $N^{3}$, the cube of the system size. This could reduce the usefulness of this approach in calculating intermolecular forces for larger systems. However, we expect that statistical errors in the energies given by such calculations might be greatly reduced by an effective implementation of correlated sampling ${ }^{7}$ procedures.

${ }^{1}$ J. Noga and W. Kutzelnigg, J. Chem. Phys. 101, 7738 (1994).

${ }^{2}$ B. Liu and A. D. McLean, J. Chem. Phys. 59, 4557 (1973).

${ }^{3}$ F. B. van Duijneveldt, J. G. C. M. van Duijneveldt-van de Rijdt, and J. H. van Lenthe, Chem. Rev. (Washington, D.C.) 94, 1873 (1994).

${ }^{4}$ S. F. Boys and F. Bernardi, Mol. Phys. 19, 553 (1970).

${ }^{5}$ I. Mayer, A. Vibok, G. Halasz, and P. Valiron, Int. J. Quantum Chem. 57, 1049 (1996).

${ }^{6}$ G. Calderoni, F. Cargnoni, A. Famulari, and M. Raimondi, J. Phys. Chem. A 106, 5521 (2002).

${ }^{7}$ B. L. Hammond, W. A. Lester, Jr., and P. J. Reynolds, Monte Carlo Methods in Ab Initio Quantum Chemistry, 1st ed. (World Scientific, Singapore, 1994).

${ }^{8}$ J. B. Anderson, J. Chem. Phys. 63, 1499 (1975).

${ }^{9}$ J. B. Anderson, J. Chem. Phys. 65, 4121 (1976).
${ }^{10}$ P. J. Reynolds, D. M. Ceperley, B. J. Alder, and W. A. Lester, Jr., J. Chem. Phys. 77, 5593 (1982).

${ }^{11}$ C. J. Umrigar, M. P. Nightingale, and K. J. Runge, J. Chem. Phys. 99, 2865 (1993)

${ }^{12}$ J. B. Anderson, C. A. Traynor, and B. M. Boghosian, J. Chem. Phys. 99, 345 (1992).

${ }^{13}$ C. Huiszoon and M. Caffarel, J. Chem. Phys. 104, 4621 (1996).

${ }^{14}$ J. B. Anderson, J. Chem. Phys. 115, 4546 (2001).

${ }^{15} \mathrm{~J}$. B. Anderson (unpublished).

${ }^{16}$ A. Bhattacharya and J. B. Anderson, Phys. Rev. A 49, 2441 (1994).

${ }^{17}$ V. Mohan and J. B. Anderson, J. Chem. Phys. 92, 6971 (1990).

${ }^{18}$ A. Bhattacharya and J. B. Anderson, J. Chem. Phys. 100, 8999 (1994).

${ }^{19}$ D. L. Diedrich and J. B. Anderson, Science 258, 786 (1992); J. Chem. Phys. 100, 8089 (1994).

${ }^{20}$ K. E. Riley and J. B. Anderson, J. Chem. Phys. 118, 3437 (2003).

${ }^{21}$ M. Mella, G. Morosi, and D. Bressanini, J. Chem. Phys. 112, 1063 (2000).

${ }^{22}$ G. W. F. Drake, M. M. Cassar, and R. A. Nistor, Phys. Rev. A 65, 054501 (2002).

${ }^{23}$ R. E. Lowther and R. L. Coldwell, Phys. Rev. A 22, 14 (1980).

${ }^{24}$ J. Komasa and J. Rychlewski, Chem. Phys. Lett. 249, 253 (1996).

${ }^{25}$ J. Komasa and J. Rychlewski, Mol. Phys. 91, 909 (1997).

${ }^{26}$ T. van Mourik, R. J. Vos, J. H. van Lenthe, and F. B. van Duijneveldt, Int. J. Quantum Chem. 63, 805 (1997).

${ }^{27}$ R. J. Gdanitz, Mol. Phys. 96, 1423 (1999).

${ }^{28}$ H. Partridge and C. H. Bauschlicher, Mol. Phys. 96, 705 (1999).

${ }^{29}$ J. van de Bovenkamp and F. B. van Duijneveldt, J. Chem. Phys. 110, 11141 (1999).

${ }^{30}$ T. Korona, H. L. Williams, R. Bukowski, B. Jeziorski, and K. Szalewicz, J. Chem. Phys. 106, 5109 (1997).

${ }^{31}$ P. C. Stancil, S. Lepp, and A. Dalgarno, Astrophys. J. 458, 401 (1996).

${ }^{32}$ F. A. Gianturco, E. Buonomo, S. Kumar, N. J. Clarke, M. Sironi, and M. Raimondi, Chem. Phys. 231, 350 (1998).

${ }^{33}$ B. K. Taylor and R. J. Hinde, J. Chem. Phys. 111, 973 (1999).

${ }^{34}$ B. K. Taylor and R. J. Hinde, Abstr. Pap.-Am. Chem. Soc. 218, 212 (Phys., Part 2) (1999).

${ }^{35}$ F. A. Gianturco, F. Paesani, R. Curik, G. Delgado-Barrio, T. GonzalezLezana, S. Miret-Arres, and P. Villarreal, Chem. Phys. Lett. 311, 255 (1999).

${ }^{36}$ E. Bodo, F. A. Gianturco, R. Martinazzo, F. Paesani, and M. Raimondi, J. Chem. Phys. 113, 11071 (2000)

${ }^{37}$ J. Komasa, W. Cencek, and J. Rychlewski, in Computational Methods in Science and Technology (Scientific Publishers OWN, 1996), Vol. 2, pp. 87-100.

${ }^{38}$ K. E. Schmidt and J. W. Moskowitz, J. Chem. Phys. 93, 4172 (1990).

${ }^{39}$ M. J. Frisch et al., GAUSSIAN 98, Gaussian, Inc., Pittsburgh, PA, 1998.

${ }^{40}$ B. Liu, Kiyosi O-Ohata, and K. Kirby-Docken, J. Chem. Phys. 67, 1850 (1977).

${ }^{41}$ E. Clementi and C. Roetti, At. Data Nucl. Data Tables 14, 177 (1974).

${ }^{42}$ D. Bressanini, G. Morosi, and M. Mella, J. Chem. Phys. 116, 5345 (2002). 